\title{
Dynamic Feedback Between a Continentlike Raft and Thermal Convection
}

\author{
ShIJIE ZHONG AND MiChaEL GURNIS
}

\author{
Deparment of Geological Sciences, University of Michigan, Ann Arbor
}

\begin{abstract}
Seismic observations of the mantle, which include long-wavelength structure, a $k^{-1}$ dependence of heterogeneity on harmonic $k$, and a heterogeneous upper boundary layer, and supercontinent kinematics may be explained by the dynamic interaction between a continent like raft and thermal convection. We have formulated finite element models of convection with rafts simulating continental plates in a cylindrical geometry. The aximuthal interconnectivity of this geometry is vital to resolve the two-way dynamics between rafts and convection. Computations show that (1) raft motion is periodic, (2) long-wavelength themal structure is significant within both thermal boundary layers and the fluid interior, and (3) the largescale thermal structure with 2 wavelength longer than the width of raft is responsible for raft motion. These three results, which are observed for a range of Rayleigh numbers, internal heating rates, and nft sizes, are a direct consequence of the dynamic interaction between the raft and convection. The physical processes for a model with a Rayleigh number of $10^{5}$ are representative: when the raft is stationary, due to the less efficient heat transfer through the raft and instabiliries from the bottom boundary layer, heat accumulates beneath the raft and results in long-wavelength thermal anomalies. The long-wavelength themal anomalies enhance raft motion. Accompanying the enhanced raft movement, the long-wavelength thermal anomalies diminish and the raft velocity decreases or the raft comes to rest. Since convection models without rafts generate less long-wavelength heterogeneity compared to the models with rafts, or continental plates, we suspect that continental plates may play a crucial role in mantle dynamics. Interestingly, raft motion with a period of about 10 transit times is usually significant; 10 transit times is about 600 m.y. if scaled to the Earth. This is close to the observed 300-500 m.y. period of supercontinent aggregation and dispersal.
\end{abstract}

\section{INTRODUCTION}

Over the last few years, oceanic plates have been successfully introduced into simple numerical models of thermal convection. Some models have introduced oceanic plates via piecewise constant velocity boundary conditions [Davies, 1988a, b], while in other models, imposed weak zones have been used to simulate plate margins [Davies, 1989]. Although these models are not dynamically selfconsistent, they have been able to predict the form of both oceanic bathemetry and heat flow [Davies, 1988a, b, 1989]. These convection models have all been steady state, but it would be of great interest to formulate truly dynamic and timedependent models of plate-mantle interaction. An important attempt to simulate time-dependent plate-mantle interactions was made by Gurnis and Hager [1988], who simulated the evolution of an oceanic plate; the resulting subducted slab showed first order changes in structure and dip as a function of time which were successfully related to the structure of seismic Benioff zones. But there are even larger spatial scale and longer time scale features of the solid Earth which are almost certainly related to plate-mantle interactions but have yet to be realistically addressed by geodynamicists. These features include: supercontinent aggregation and dispersal, first-order fluctuations in sea level, and periodicity in the age of crustal rocks.

A variety of studies [Hoffman, 1992] suggest that supercontinents may have repeatedly formed and then

Copyright 1993 by the American Geophysical Union.

Paper number 93JB00193.

0148-0227/93/93JB-00193\$05.00 dispersed during the Phanerozoic and late Proterozoic. The final assembly of Pangea by the late Triassic is well known [Smith et al., 1981], although its assembly is still not well constrained. Pangea formed with the collision of the North American/European, Gondwana, and Siberian block, about 200 m.y. ago. Gondwana (composed of South America, Africa, India, Australia, and Antarctica) was a large coherent continent since the late Proterozoic. Once formed, Pangea started to breakup within 50 m.y. [Smith et al., 1981]. Before the Carboniferous, paleomagnetic, paleoclimatological, and biogeographical patterns suggest that Laurentia and Gondwana assembled into a large continental mass by the collision of northwest Africa into the eastern United States in the Late Silurian/Early Devonian [Miller and Kent, 1988; Van der Voo, 1988]. Miller and Kent [1988] refer to this Early Devonian assemblage as a supercontinent, but because the paleopoles of the Baltic Shield and Russian Platform are poorly known at this time [Van der Voo, 1988], it may be premature to refer to it as a supercontinent. The Early Devonian assemblage only persisted for tens of millions of years. There has been considerable speculation about an Eocambrian supercontinent [Bond et al., 1984; Van der Voo et al., 1984; Dalziel, 1991; Hoffman, 1991]. The rapid subsidence of the Paleozoic miogeocline of the southem Canadian Rocky mountains [Bond and Kominz, 1984] as well as similar subsidence in eastem North America, Argentina, Australia, and elsewhere suggest the development of a passive margin nearly as long as that which formed after the breakup of Pangea [Bond et al., 1984]. Bond et al. [1984] suggested that this is consistent with the breakup of an Eocambrian supercontinent between 625 and $555 \mathrm{Ma}$. This conclusion is supported by a first-order rise in relative sea level in the early Paleozoic [Hoffman, 1992], similar to that in the Creataceous following the breakup of 
Pangea. All of these studies suggest that supercontinent motion is aperiodic, with a period of 300 to 500 m.y. Moreover, the period over which the continent remains assembled may be short (perhaps $\sim 20 \%$ ) in comparison to the overall time period from breakup to breakup. Clearly, if mantle convection is the driving force responsible for supercontinent kinematics, then such continental kinematics demonstrates that mantle convection has not been in a steady state over the last 1 b.y.

Quite distinct from geologic observations which sense how the mantle changes over long time periods are geophysical observations which sense what Earth looks like at a large scale. Recent seismic tomography studies have resolved threedimensional seismic velocity anomalies throughout the upper and lower mantle. The seismic velocity anomalies have been regarded as variations in mantle temperature, although seismic velocities change with composition as well. By mapping the seismic velocity anomalies, these studies almost certainly will help us understand Earth dynamics. Early studies used $P$ wave travel time data reported by the International Seismology Centre (ISC) to image mantle structure. Dziewonski et al. [1977] and Dziewonski [1984] parameterized the lower mantle with lower order harmonics, while Hager and Clayton [1989], and Inoue et al. [1990] represented the whole mantle with distinct cells. Tanimoto [1990a] utilized long-period $S H$ body wave and long-period Love wave data to obtain $S$ wave velocity structure within the whole mantle. Although the methods and data are different, all these studies have yielded some common features of mantle structure, such as fastvelocity ariomalies in the lower mantle surrounding the Pacific Ocean. More importantly, these studies have consistently displayed significant spatial power at the gravest spherical harmonics from degrees 1-6. For example, Tanimoto [1990a] demonstrated that the power at harmonic degree 2 was dominant through the whole mantle except for a layer at 1000 $1300 \mathrm{~km}$ depth. Considering that ISC data used in P-wave tomography inversions may contain significant random errors, Gudmundsson [1989] and Davies [1990] designed stochastic inversion methods in which the variation of lateral heterogeneity with depth was obtained. They showed that even when the power of spatial variability within the lower mantle was expanded up to harmonic degree 25 , the power was dominated by the lower harmonic degrees, having a maximum at degree 1 and decreasing rapidly with harmonic degree.

Even without inverting for structure, Woodward and Masters [1991] and $S u$ and Dziewonski [1991] showed that the longwavelength heterogeneity was observed from raw travel time residue data. By analyzing $S c S-S$ differential travel times, Woodward and Masters [1991] specifically showed that the three dimensional (3-D) structure within the lower mantle was dominated by continental-scale features. This conclusion is rather convincing, because $S c S-S$ differential travel times are insensitive to upper mantle structure. Based on studying long period SS travel time anomalies, $S_{u}$ and Dziewonski [1991] demonstrated that power of the travel time residues was all accumulated at harmonic degrees less than 6 , although the data were expanded up to harmonic degree 36 . They concluded that mantle heterogeneity had a wavelength over $6000 \mathrm{~km}$.

Based on their surface wave studies, Tanimoto [1990b] and Zhang and Tanimoto [1991] showed that spectra or root-meansquare (RMS) of Love wave phase velocity at a period of $100 \mathrm{~s}$ is concentrated at low harmonic degrees, $k$, and decreases rapidly with $k$ approximately as $k^{-1}$. Since surface waves mainly contain information about shallow parts of Earth, this $k^{-1}$ dependence has been explained as an outcome of largescale features on Earth's surface, such as the existence of continents [Tanimoto, 1990b]. While the long-wavelength heterogeneity dominates mantle structure, its power varies greatly with depth, having peaks at both the shallow part of the mantle and core-mantle boundary (CMB) [Gudmundsson, 1989; Tanimoto, 1990b]. This indicates that CMB and Earth surface may be more heterogeneous. Moreover, the power of heterogeneity is greater at the shallow part of the mantle than that in CMB [Gudmundsson, 1989; Tanimoto, 1990b].

Such large-scale mantle heterogeneity should be an outcome of mantle convection models. Jarvis and Peltier [1986, 1990] have studied the spectral decomposition of the temperature field in Cartesian convection models with constant viscosity and reflecting boundary conditions. They showed that heterogeneity with a wavelength comparable to the circulation length was significant within thermal boundary layers and that the power of the heterogeneity was greater in thermal boundary layers compared to the fluid interior. In a similar convection model, Honda [1987] used the root-mean-square of temperature as a measure of heterogeneity and found that more heterogeneity occurred in thermal boundary layers. These results about thermal boundary layers seem to be consistent with seismic tomography [Tanimoto, 1990b]. Outside of thermal boundary layers, the long-wavelength thermal structure was found to be insignificant for models with aspect ratio ranging from 1 to 3 [Jarvis and Peltier, 1986, 1990]. Machetel [1990] showed that for an axisymmetric convection model with relatively realistic parameters of Earth, the thermal structure was dominated by short-wavelength anomalies. Faced with a contradiction between the results from his models and seismic tomography, Machetel [1990] suggested that the long-wavelength structure observed from seismic tomography might be artifacts resulting from truncation. Machetel further displayed that after truncation, the short-wavelength thermal anomalies from his models would yield long-wavelength structure apparently compatible with tomographic mantle anomalies. However, using the seismic velocity anomalies as driving sources in a viscous model, Hager and Clayton [1989] were able to explain about $90 \%$ of the long-wavelength geoid. With a similar approach, Ricard and Vigny [1989] showed that current plate velocities can also be successfully explained. These two studies suggest that the observed seismic longwavelength anomalies may indeed be real features of the mantle.

What does not seem to be well appreciated is that the present state of the mantle is an integral of mantle convection over time. Indeed, the presently observed large-scale heterogeneity of the mantle may be a fundamental byproduct of large-scale, time-dependent mantle convection as demanded by the geologic observations reviewed above. A successful mantle convection model must not only explain the origin of the large-scale heterogeneity observed with tomography, geoid, present plate motions, bathymetry, and heat flow, but it must also be consistent with the long-term trends observed in the geologic record, like supercontinent aggregation and dispersal.

Why did the convection models of Machetel [1990] fail to generate large-scale heterogeneity? One possibility is a lack of plates. Although oceanic plates are the surface manifestation of convection (i.e., are the outer mobile thermal boundary of mantle convection), continental plates can act as 
insulating lids [Elder, 1976] and thus impose a large-scale heterogeneity on the Earth's surface. This heterogeneity could play an important role in determining Earth's thermal structure [Elder, 1976; Anderson, 1982]. The reflecting boundary condition and the small aspect ratio of the box in the Jarvis and Peltier [1986, 1990] models may make their models unsuitable to address large-scale features within the mantle. Unlike typical convection models with Cartesian geometry where the vertical boundaries of the box are loaded, the geometry of the mantle allows material to freely move laterally. Such interconnectedness could have a profound effect on the time dependence of mantle flow. The spherical geometry of the mantle may also have significant effect on convection. Without proper considerations of these essential features, a convection model may be incapable of generating large-scale structure which has been observed within the mantle.

Lateral interconnectedness and continental plates were explored by Gurnis [1988] with a finite element method in a Cartesian geometry with periodic boundary conditions. Although this model only included the subducting feature of oceanic plates by prescribing two weak margins on the periphery of a raft, the raft properly simulated a continent, being mechanically stiffer, thicker, and less efficient in heat transfer than the normal boundary layer. In this model, heat accumulates beneath the raft and results in a large extensional force on the raft, thus dispersing the raft and moving the split rafts off the hot zone. Since periodic boundary conditions were used, the flow often made the split rafts collide. This model provides a plausible model for the repeated occurrence of dispersal and collision of supercontinents. Using an identical technique to introduce rafts in a cylindrical annulus, Gurnis and Zhong [1991] (hereafter referred to as paper 1) showed that outside the thermal boundary layers significant power at the gravest harmonics could be generated and the heterogeneity was strongly correlated with raft motion. Paper 1 also showed that both the less efficient heat transfer through the raft and plume-plume collisions are the processes most responsible for the generation of the power at the gravest harmonics. However, in paper 1 , only thermal structure at wavenumber 1 was investigated in an effort to relate raft motion to longwavelength thermal structure. It is essential to study how other long-wavelength structure interacts with rafts. There were also two simplifications in paper 1: the lack of internal heating and a relatively low Rayleigh number. Several numerical studies [McKenzie et al., 1974; Davies, 1986] have shown that internal heating has considerable influence on the form of convection, including the thickness of the thermal boundary layers and the width of plumes. Higher Rayleigh number also affects convection due to more frequent thermal boundary layer instabilities [McKenzie et al., 1974]. Here we will study the interaction between large-scale thermal structure of different wavelength and rafts and the effects of internal heating and more realistic Rayleigh numbers.

We will systematically present a series of numerical models in which internal heating, Rayleigh number, and raft size are varied. The results consistently show that raft motion is aperiodic and that significant large-scale lateral heterogeneity exists within and outside thermal boundary layers. In addition, the lateral heterogeneity with a wavelength larger than the raft width is strongly correlated with raft motion. The results also show that the continent like raft is important for developing the long-wavelength structure.

\section{Governing Equations And Finte Element ANalysis in A Cylndrical Geometry}

\subsection{Governing Equations}

The governing equations for mantle convection are derived from the conservation of mass, momentum and energy. Since the mantle has a very high Prandtl number, the inertial terms in the momentum equation are negligible. With the Boussinesq approximation, the fluid is effectively incompressible [McKenzie et al., 1974; Jarvis and Peltier, 1982]. The momentum, continuity, and energy equations become, respectively:

$$
\begin{gathered}
\mu \nabla^{2} \vec{u}=-\nabla P+\rho_{o} \alpha\left(T-T_{o}\right) g \vec{e}_{r}, \\
\nabla \cdot \vec{u}=0, \\
\frac{\partial T}{\partial t}+\vec{u} \cdot \nabla T=\kappa \nabla^{2} T+H,
\end{gathered}
$$

where all the differential operators are in a cylindrical coordinate $(r, \theta)$ system $(\theta$ and $r$ are the azimuthal and radial coordinates, respectively), $\vec{u}=\left(u_{r}, u_{\theta}\right)$ is the velocity, $T$ is the temperature, $P$ is the pressure, $H$ is the heat source, $P_{0}$ and $T_{0}$ are the reference density and temperature, respectively, $K$ is the thermal conductivity, $\mu$ is the dynamic viscosity, $g$ is the gravitational acceleration, $\alpha$ is the coefficient of thermal expansion, $\vec{e}_{r}$ is the unit vector in the radial direction, and $t$ is the time. The boundary conditions utilized here are free-slip and isothermal.

$$
\begin{gathered}
\left.T\right|_{r=R_{o}=T_{o},\left.\quad T\right|_{r=R_{i}}=T_{i},} \\
\left.u_{r}\right|_{r=R_{o}}=\left.u_{r}\right|_{r=R_{i}}=\left.\frac{\partial\left(u_{\theta} / r\right)}{\partial r}\right|_{r=R_{o}}=\left.\frac{\partial\left(u_{\theta} / r\right)}{\partial r}\right|_{r=R_{i}}=0,
\end{gathered}
$$

where $T_{o}$ and $T_{i}$ are temperature on the outer (radius $R_{0}$ ) and the inner (radius $R_{i}$ ) boundaries, respectively. The equations are nondimensionalized in a cylindrical coordinate through introduction of the following characteristic scales (primed variables are dimensionless):

$$
\begin{gathered}
r=R_{o} r^{\prime} ; \quad T=\left(T_{i}-T_{o}\right) T^{\prime}+T_{o} ; \quad \gamma=H D^{2} / \mathrm{K} ; \\
t=t^{\prime} U_{o} / R_{o} ; \quad\left(u_{r}, u_{\theta}\right)=U_{o}\left(\dot{u_{r}}, u_{\theta}^{\prime}\right) ; \quad U_{o}=\kappa / R_{o} ; \\
P=P^{\prime} R_{o}^{2} / \mathrm{\kappa},
\end{gathered}
$$

where $D$ is the thickness of the fluid layer. The normalization of $r$ by $R_{o}$ makes the radius of outer boundary 1 .

Substitution of (6) into (1)-(5) yields

$$
\begin{gathered}
\nabla^{2} \vec{u}=-\nabla P+\zeta^{-3} R_{a T e_{r},} \\
\nabla \cdot \vec{u}=0, \\
\frac{\partial T}{\partial t}+\vec{u} \cdot \nabla T=\nabla^{2} T+\zeta^{-2} E,
\end{gathered}
$$

where

$$
\begin{gathered}
\zeta=D / R_{o}, \\
R a=\frac{\rho_{o g} \alpha D^{3}\left(T_{i}-T_{o}\right)}{\kappa \mu}
\end{gathered}
$$




$$
E=\frac{\gamma}{T_{i}-T_{0}}
$$

The boundary conditions become

$$
\begin{gathered}
\left.\left.T\right|_{r=1=0,} T\right|_{r=1-\zeta=1,} \\
\left.u_{r}\right|_{r=1}=\left.u_{r}\right|_{r=1-\zeta}=\left.\frac{\partial\left(u_{\theta} / r\right)}{\partial r}\right|_{r=1}=\left.\frac{\partial\left(u_{\theta} / r\right)}{\partial r}\right|_{r=1-\zeta}=0,
\end{gathered}
$$

where the primes have now been dropped for simplicity.

$\zeta$ is the ratio of the fluid layer thickness to the radius of outer boundary. $\zeta$ has been set to 0.5 for all calculations and is close to the value for whole mantle convection. Unlike the simple case of internal heating with a prescribed bottom heat flux [McKenzie et al., 1974], neither the Rayleigh number can be conveniently defined in terms of the heat transferred out of the surface when convection is absent, nor the internal heating parameter, E, can be regarded as the ratio of internal to total heating or the internal heating rate. Since the heat flux into the bottom can not be a priori determined for the prescribed isothermal condition, the internal heating rate can only be obtained after the above equations are solved and the heat fluxes through both the bottom and top boundaries are determined. For time dependent models, averaged internal heating rate through time, $\xi$, is proper to be used to characterize models and is defined as

$$
\xi=\int_{0}^{t}\left[q_{t}-(1-\zeta) q_{b}\right] d t^{\prime} / \int_{0}^{t} q_{t} d t^{\prime}
$$

where $q_{t}$ and $q_{b}$ are heat fluxes through top and bottom boundaries, respectively.

The initial condition used for all models is

$$
T=\frac{\ln (r)}{\ln (1-\zeta)}-\varepsilon \cos \left(k_{0} \theta\right) \sin (2 \pi r)
$$

where $k_{0}$ is the perturbation wavenumber, and $\varepsilon$ is the magnitude of the perturbation. In our models, $k_{0}$ and $\varepsilon$ are set to 2 and 0.01 , respectively.

\subsection{Finite Element Analysis}

The finite element technique is the same as that adopted in the original Cartesian geometry version of the finite element code [King et al., 1990]. Appropriate modifications have been made in the finite element analysis for the cylindrical formulation.

A penalty formulation is used to solve the momentum equation with the incompressibility constraints [Hughes, 1987]. The element stiffness matrix $k^{e}$ can be written as

$$
k^{e}=\left[k_{p q}^{e}\right]
$$

where

$$
k_{p q}^{e}=e_{i}^{T}\left[\int_{V^{e}} B_{a}^{T}\left(D_{\lambda}+D_{\mu}\right) B_{b} d V\right] e_{j}=e_{i}^{T} k_{a b}^{e} e_{j}
$$

where $D_{\lambda}$ is the matrix representing the penalty; $D_{\mu}$ is the material properties matrix [Hughes, 1987]; the superscripts $T$ represent transpose of a matrix; $e_{i}$ and $e_{j}$ are the unit direction vectors; indices $a$ and $b$ are elemental node indices; and $B_{a}$ in a cylindrical coordinate system, is

$$
B_{a}=\left[\begin{array}{cc}
\frac{N_{a, \theta}}{r}, & \frac{N_{a}}{r} \\
0, & N_{a, r} \\
N_{a, r}-\frac{N_{a}}{r}, & \frac{N_{a, \theta}}{r}
\end{array}\right],
$$

where $N_{a}$ is the shape function at the node index $a$, and $N_{a, i}$ is the derivative of $N_{a}$ with respect to the coordinate $i$. In terms of $B_{a}, k_{a b}^{e}$ can be written as the sum of $k_{\lambda}$ and $k_{\mu}$, where

$$
k_{\lambda}=\int_{V^{e}} B_{a}^{T} D_{\lambda} B_{b} d V, \quad k_{\mu}=\int_{V^{e}} B_{a}^{T} D_{\mu} B_{b} d V .
$$

A one-point Gaussian quadrature is adopted to evaluate $k_{\lambda}$ and four-point Gaussian quadrature is used to evaluate $k_{\mu}$ [Hughes, 1987].

The energy equation is solved with a streamline upwind Petrov-Galerkin method [Brooks, 1981]. The weak form of the equation is given by

$$
\begin{gathered}
\int_{V}(w+p) \frac{\partial T}{\partial t} d V=-\int_{V}(w+p)\left(u_{i} T_{, i}-E\right) d V \\
-\int_{V} w_{, i} T_{, i} d V+\int_{\Gamma_{h}} w T_{, i} n_{i} d T,
\end{gathered}
$$

where $\Gamma_{h}$ is a boundary prescribed heat flux, $w$ is the standard weighting function, and $(w+p)$ is the Petrov-Galerkin weighting function with $p$, the discontinuous streamline upwind part. In cylindrical coordinates,

$$
\begin{aligned}
& w_{, i} T_{, i}=\frac{\partial w}{\partial r} \cdot \frac{\partial T}{\partial r}+\frac{1}{r^{2}} \frac{\partial w}{\partial \theta} \cdot \frac{\partial T}{\partial \theta}, \\
& p=k^{\prime} \frac{1}{u_{r}^{2}+u_{\theta}^{2}}\left(u_{r} \frac{\partial w}{\partial r}+\frac{u_{\theta}}{r} \cdot \frac{\partial w}{\partial \theta}\right),
\end{aligned}
$$

where $K^{\prime}$ is an artificial diffusivity.

In order to assure that the code is operating properly, a comparison has been made between critical Rayleigh numbers from a linear stability analysis and those obtained from the finite element code. The comparison has been made for isoviscous flows with isothermal and free-slip boundary conditions (Appendix A).

A raft is incorporated into the flow with a technique that was developed for a Cartesian flow [Gurnis, 1988]. Within the Eulerian finite element mesh, a region is assigned a viscosity $10^{3}$ times the background viscosity, and this region represents the raft. Two weak margins having a viscosity 0.1 times the background are on the raft periphery. The background is isoviscous. The high viscosity of the raft and the low viscosity in the two margins result in "plate like" behavior. With steady state models, King and Hager [1990] showed that there is no significant change in the solution when the raft viscosity was increased to $10^{5}$ times the background viscosity or when the margin viscosity was decreased to 0.01 times the background viscosity. The center node of the raft is pinned to form a reference frame (Figure 1). With respect to this pinned node, there can exist a nonzero, mean velocity in the azimuthal, $\theta$, direction. The velocity represents the motion of 


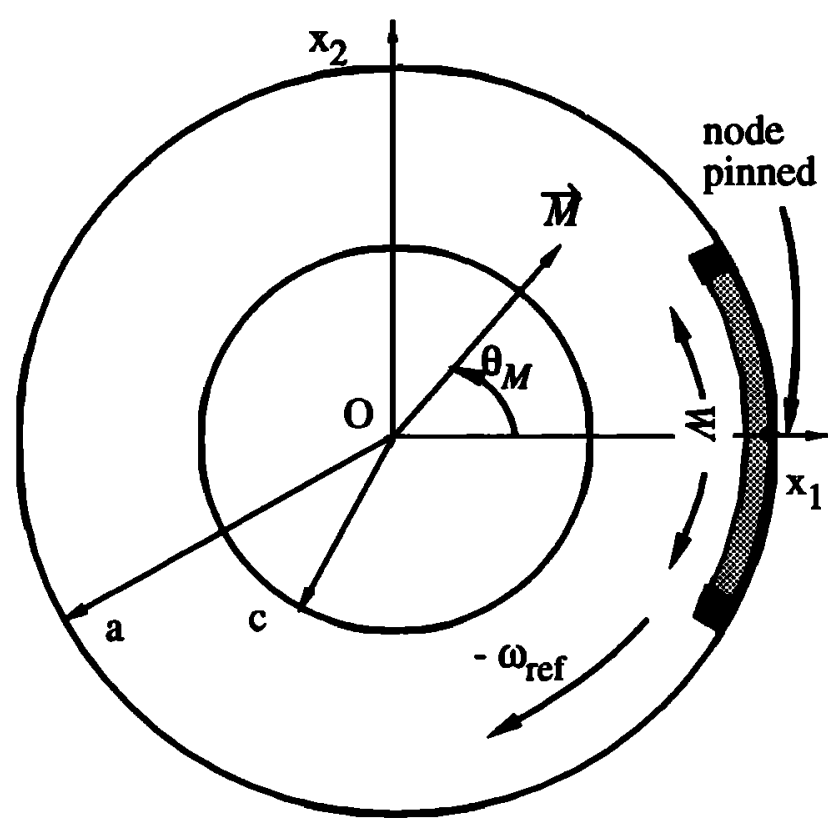

Fig. 1. Setup of the thermal convection model with a continent like raft in a cylindrical geometry. $a=1.0 ; c=0.5$; thickness of the raft and weak margins is 0.0625 ; width of the weak margins is 0.1 ; width of the raft $W$ varies from case to case (Table 1); other parameters are defined in text.

the pinned node with respect to the bulk of the fluid. The frame of reference of the system is transformed by first determining the mean azimuthal velocity

$$
\omega_{\text {ref }}=\frac{1}{S} \int_{S} \frac{u_{\theta}}{r} d S^{\prime},
$$

where the integration domain $S$ is the whole fluid layer, and then determining the azimuthal offset $\theta_{\text {ref }}$ between the pinned node and center of mass as a function of time:

$$
\theta_{\text {ref }}=\int_{0}^{t} \omega_{\text {ref }} d t^{\prime},
$$

During postprocessing, visualizations of the temperature can be created by adding $-\theta_{\text {ref }}$ to the azimuthal coordinate of each node.

For all the cases with $R a=10^{5}$, numerical tests showed that a mesh with $240 \times 32$ elements was sufficient to resolve the thermal boundary layers. When $R a=10^{6}$, both boundary layers and plumes become thin, as a result, sufficient elements are required. For these cases, $360 \times 40$ elements are used, and in the radial direction, the mesh is refined so that better resolution is acquired. We have observed that there are more than four elements to resolve the top and bottom thermal boundary layers even when internal heating is significant.

\section{Measures of Time and Spatial Dependencies}

In order to understand raft motion, long-wavelength heterogeneity, and their time dependencies, several measures have been made. First, the temperature field has been deconvolved into its frequency components:

$$
\bar{T}_{k}=\frac{1}{N} \sum_{n=0}^{N-1} \frac{1}{\left(r_{2}-r_{1}\right)} \int_{\eta}^{m} T(r, n \Delta \theta) d r \cdot \exp \left(-i \frac{2 \pi n}{N} k\right) \text {, }
$$

where $\bar{T}_{k}$ is the azimuthal Fourier transform of the temperature averaged radially between $r_{1}$ and $r_{2}$. While dealing with heterogeneity within the fluid interior, $r_{1}$ and $r_{2}$ equal 0.625 and 0.875 , respectively. But while dealing with heterogeneity within thermal boundary layers, $r_{1}$ and $r_{2}$ vary with $R a$, only sampling temperature within boundary layers. Second, a first moment of density distribution is defined as

$$
\begin{gathered}
\vec{M}=\int_{S} \rho x_{i} d S^{\prime} \cdot \bar{e}_{i} / \int_{S} \rho d S^{\prime}, \\
p=p_{0}\left[1-\alpha\left(T-T_{0}\right)\right],
\end{gathered}
$$

where the components $\vec{e}_{i}$ are in a Cartesian system (Figure 1) in which the origin is identical to the origin in the cylindrical system and the vector pointing to the center of the raft defines the $x_{I}$ axis. It is easy to show that the orientation and relative magnitude of $\vec{M}$ are independent to $\alpha$. $\vec{M}$ measures the phase offset between the position of the raft and the main cold anomalies, which is helpful in understanding the interaction between raft motion and thermal structure. In plots presented here, at each time $\bar{M}$ is represented by an arrow, and orientation of the arrow is determined by fixing the raft at the right end of a time axis. Third, heat flux out of the raft, which is utilized to study the temperature field beneath the raft. The calculation of heat flux on boundaries is similar to that given by Ho-Liu et al. [1987]. Nondimensional heat flux can be expressed as

$$
q=u_{r} T-\frac{d T}{d r},
$$

$q$ is first evaluated in each boundary element, and the computed elemental heat flux is then projected to boundary nodes by using the standard smoothing approach. Fourth, a depth dependence of root-mean-square (RMS) of temperature field is used to measure the heterogeneity at different depths. Following Honda [1987], the definition of RMS is

$$
<\delta T(r)>=\sqrt{\frac{1}{2 \pi} \int_{0}^{2 \pi}\left[T(r, \theta)-T_{m}(r)\right]^{2} d \theta .}
$$

where $T_{m}(r)$ is the mean temperature at a radius $r$. Finally, noticing that the diffusion time during which a process elapses varies with Rayleigh number, it is proper to measure the process in terms of a transit time which is the time a parcel of fluid taken to traverse the fluid depth with the average velocity on the top surface, or

$$
t_{\text {transit }}=\int_{0}^{t} \frac{\overline{\bar{u}_{\theta}(1, t)}}{\zeta} d t
$$

where

$$
\bar{u}_{\theta}\left(1, t^{\prime}\right)=\frac{1}{2 \pi} \int_{0}^{2 \pi}\left|\mu_{\theta}\left(1, \theta, t^{\prime}\right)\right| d \theta
$$

is the average absolute velocity on the top at diffusion time $t$ '. Our computations show that transit always varies linearly with $t$, as a result, (29) can be written as

$$
t_{\text {transit }}=\frac{\tilde{u}_{\theta}}{\zeta} t,
$$


where $\tilde{u}_{\theta}$ is the called characteristic velocity and can postpriori be found from the linear relationship between $t_{\text {transit }}$ and diffusion time $t$ (equation (31)).

\section{Results}

The role of Rayleigh number, raft size, and internal heating have been explored in a variety of cases (Table 1). In all cases with a raft, we find (1) periodicity in raft motion, (2) significant long-wavelength structure within and outside thermal boundary layers, and (3) a correlation between raft motion and long-wavelength thermal structure within the fluid interior. By significant long-wavelength structure, we mean that the amplitude of temperature variations for harmonic degrees from 1 to 6 is significantly greater than the amplitude at higher harmonics. In section 3.1, two cases without rafts are presented serving as calibrations, while in sections 3.2 and 3.3 cases with rafts at $R a=10^{5}$ and $R a=10^{6}$, respectively, are presented. For each case with a raft, an animation of temperature fields, spectra of temperature fields and raft velocity are presented on the accompanying video tape (Appendix B).

\subsection{Convection Without Rafts}

The calculation for free convection at $R a=10^{5}$ and no raft (case 1) shows that a steady-state is easily reached (Figure $2 a$ ). Not surprisingly, in the steady state, convection cells are equal in size in this purely bottom heated case, and the number of cells depends on the initial perturbation $k_{0}$. The spectra (Figure $2 b$ ) clearly reflect the spatial regularity of convection with significant power at discontinuous wavenumbers. These results are qualitatively similar to those of Jarvis and Peltier [1986], who dealt with a Cartesian region filled with a single cell. It is quite evident that there is no significant power at degree 1 to degree 3; the first appearance of variability at degree 4 simply corresponds to the regular spacing of cells.

When the Rayleigh number is increased to $10^{6}$ and $E$ to 10 (case 2), a steady state is not achieved. The average internal heating rate, found postpriori, is $46 \%$ (Table 1). The spectra at a single instant in time, but representative of all time (Figure 2d), show several important features. Significant longwavelength heterogeneity is found in the bottom thermal boundary layer but not in either the fluid interior or the top thermal boundary layer, implying that short-wavelength structure characterizes the top boundary layer and the fluid interior. Jarvis and Peltier [1986] found that for Cartesian convection models long-wavelength heterogeneity is equally

TABLE 1. Model Parameters and Results

\begin{tabular}{cccccccc}
\hline Case & $R a$ & $W / D$ & $E$ & $\xi_{2} \%$ & $T_{\operatorname{mean}}$ & $F_{\text {rs }}$ & $V_{\max }$ \\
\hline 1 & $10^{5}$ & 0 & 0 & 0 & 0.377 & -- & -- \\
2 & $10^{6}$ & 0 & 10 & 46 & 0.520 & -- & -- \\
3 & $10^{5}$ & 4 & 0 & 0 & 0.423 & 0.255 & 292 \\
4 & $10^{5}$ & 2 & 0 & 0 & 0.397 & 0.277 & 320 \\
5 & $10^{5}$ & 4 & 7 & 68 & 0.653 & 0.332 & 299 \\
6 & $10^{5}$ & 2 & 7 & 63 & 0.621 & 0.358 & 251 \\
7 & $10^{6}$ & 4 & 0 & 0 & 0.424 & 0.158 & 1229 \\
8 & $10^{6}$ & 2 & 0 & 0 & 0.398 & 0.162 & 1424 \\
9 & $10^{6}$ & 4 & 10 & 53 & 0.590 & 0.203 & 1156 \\
10 & $10^{6}$ & 2 & 10 & 50 & 0.558 & 0.212 & 1234 \\
\hline
\end{tabular}

$F_{r s}$ is the ratio of the temporally averaged heat flux out of the raft to the average heat flux from the surface excluding the raft. $V_{\max }$ is the maximum raft velocity for each case.
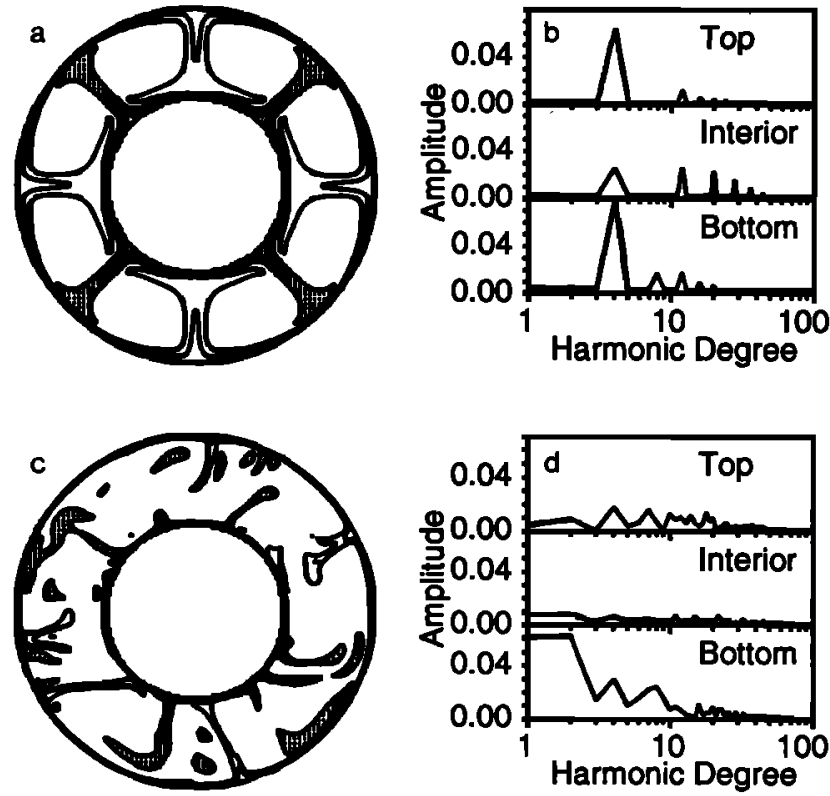

Fig. 2. Representative temperature fields and corresponding spectra for cases 1 and 2. (a) steady state temperature field for case 1; (b) apectra of the temperature field shown in a for top and bottom boundary layers and fluid interior, (c) temperature for case 2 at diffusion time 0.01441; (d) spectro of the temperature field in Figure $2 c$. In Figures $2 a$ and $2 c$, the shaded regions have a temperature greater than 0.5 and 0.6 , respectively.

significant within the top and bottom boundary layers as long as the internal heating is less than $75 \%$, a result different from ours. Such a difference may result from a difference in geometry of two sets of models. The depth-dependence of RMS (Figure 3) shows that heterogeneity in the bottom boundary layer is greater than that in the top boundary layer.

\subsection{Cases With Rayleigh Number $10^{5}$}

With no internal heating, we have shown that longwavelength heterogeneity could be generated and related to raft motion at $R a=10^{5}$ (paper 1). But in paper 1, we were not able to address in detail many phenomena including the mechanism of episodic raft motion. In this section, we systematically

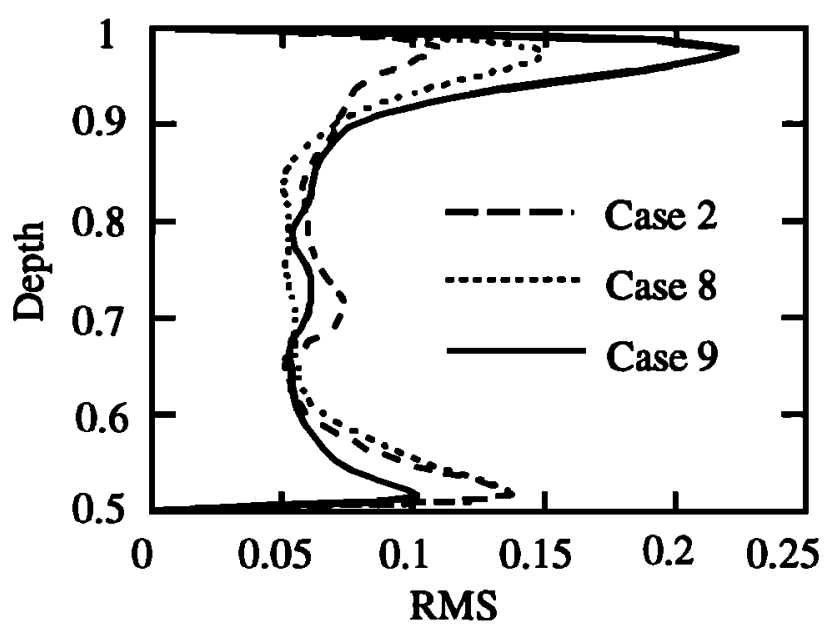

Fig. 3. Comparison between depth dependencies of the RMS of temperature. Long dashed line, short dashed line and solid line are for the temperatures shown in Figure $2 c$ (case 2), Figure 13a (case 8), and Figure $13 c$ (case 9), respectively. 
explore the effects of internal heating and raft size when $R a$ $=10^{5}$ (cases 3-6, Table 1). Although only two different raft sizes (WID $=2$ and 4$)$ are considered, these two sizes may be of great relevance to Earth. If scaled to Earth with whole mantle convection, a raft of $W / D=2$ has a length of about $6000 \mathrm{~km}$, which is the typical size for current continental plates; a raft of $W I D=4$ accounts for about $30 \%$ of the circumference of the surface, and this percentage may be close to the size of supercontinents.

For cases 3 and 4 with raft widths of 4 and 2 times the fluid layer, respectively, and only bottom heating, variations in raft size does not significantly effect the generation of longwavelength structure. Three significant features may be observed for case 3: (1) periodic raft motion $V$ (Figure 4), (2) a correlation between raft motion and long-wavelength thermal structure, including $T_{1}$ and $T_{2}$, heat flux out of the raft $F$, and first moment of the density distribution (Figure 4), and (3) dominance of long-wavelength heterogeneity within the fluid interior and thermal boundary layers (Figures 5 and 6).

We will see that time histories (Figure 4) are helpful in understanding the dynamics of convection coupled with a raft, especially for the high Rayleigh number cases, and deserve to be discussed in detail at lower Rayleigh number for reference. Time histories of $T_{1}, T_{2}, T_{3}$, and $T_{4}$ are presented for case 3 in order to understand which wavelength of thermal structure controls raft motion for a given raft size $(W / D=4)$. Raft motion correlates with $T_{1}$ and $T_{2}$ in such a way that any burst in raft motion usually coincides with a peak in either $T_{1}$, or $T_{2}$, or both (Figure 4). However, $T_{3}$ and $T_{4}$ are not simply correlated with raft motion. Since $T_{1}$ and $T_{2}$ have a wavelength longer than the raft width, and $T_{3}$ (measured at the mid-depth of the fluid layer) and $T_{4}$ have a wavelength smaller than the raft. this suggests that raft motion is controlled by a thermal structure whose wavelength is longer than the raft width.

In order to illuminate the processes controlling the largescale thermal structure and raft motion, temperature fields (Figure 5) and raft motion at four instants in time (Figure 4) are analyzed (the animation for case 3 in the accompanying video

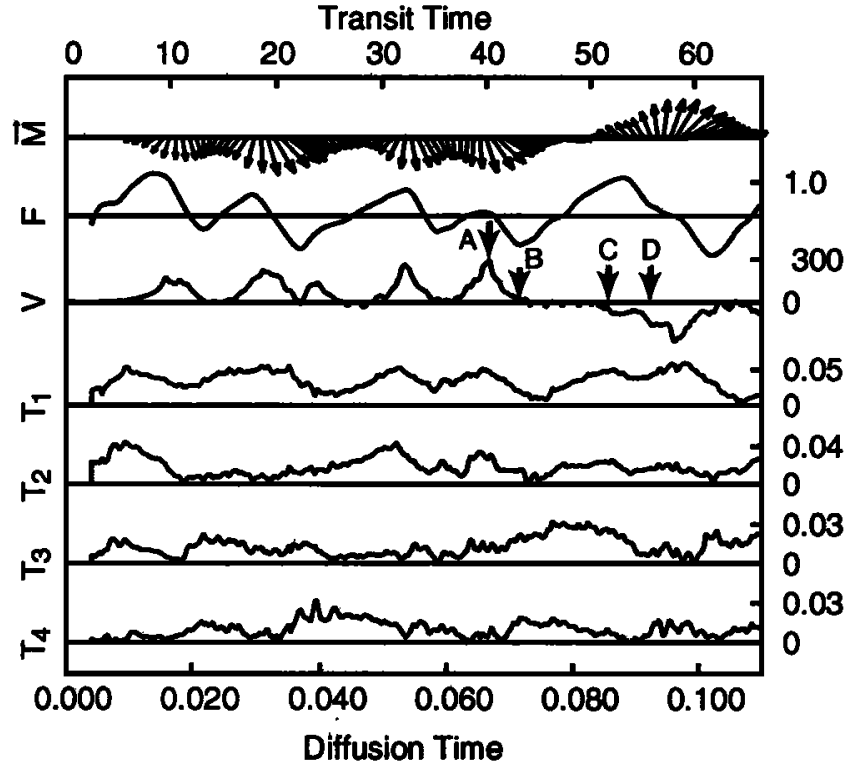

Fig. 4. Time history plot for case 3. $T_{1}, T_{2}, T_{3}$, and $T_{4}$ represent the absolute amplitude of spectra within the fluid interior at harmonic degrees $1,2,3$, and 4 , respectively. $V$ is raft velocity: $F$ is heat flux out of the raft; $\bar{M}$ is the first moment of the density distribution.
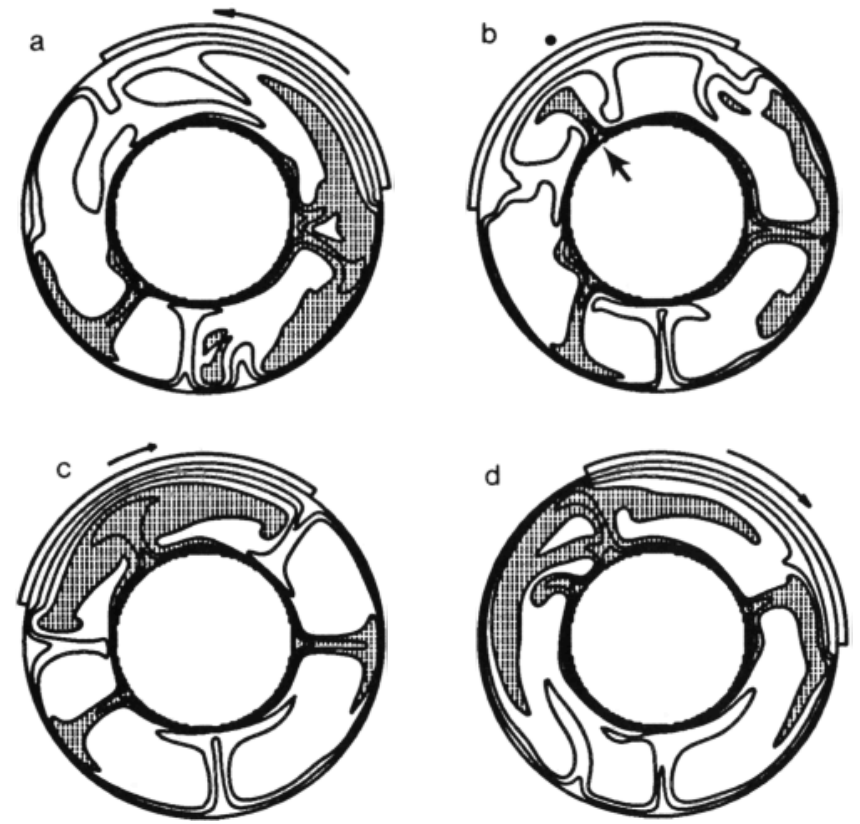

Fig. 5. Temperature field for the four instants labeled in Figure $\mathbf{4}$ for case 3. The arrows and dots shown over the raft reflect current raft velocity: dots represent negligible raft velocity and the length of the arrows represents relative magnitude of raft velocity. The other arrows point to plumes discussed in the text. The shaded regions have a temperature greater than to 0.5 .

tape also shows the physical process). At A, (Figure 4), the raft velocity, $T_{1}$, and $T_{2}$ have reached the peak amplitude, and a first moment of the density distribution, $\bar{M}$, at $90^{\circ}$ implies that the raft is located mid-way between large-scale hot and cold areas. From $A$ to B, the raft moves (Figures $5 a$ and $5 b$ ) off the hot region and approaches a downwelling, which can also be observed through the time histories (Figure 4): raft velocity and heat flux decrease and $\vec{M}$ becomes directed toward the raft center. With the slowdown of the raft, $T_{1}, T_{2}$, and $\vec{M}$ decrease
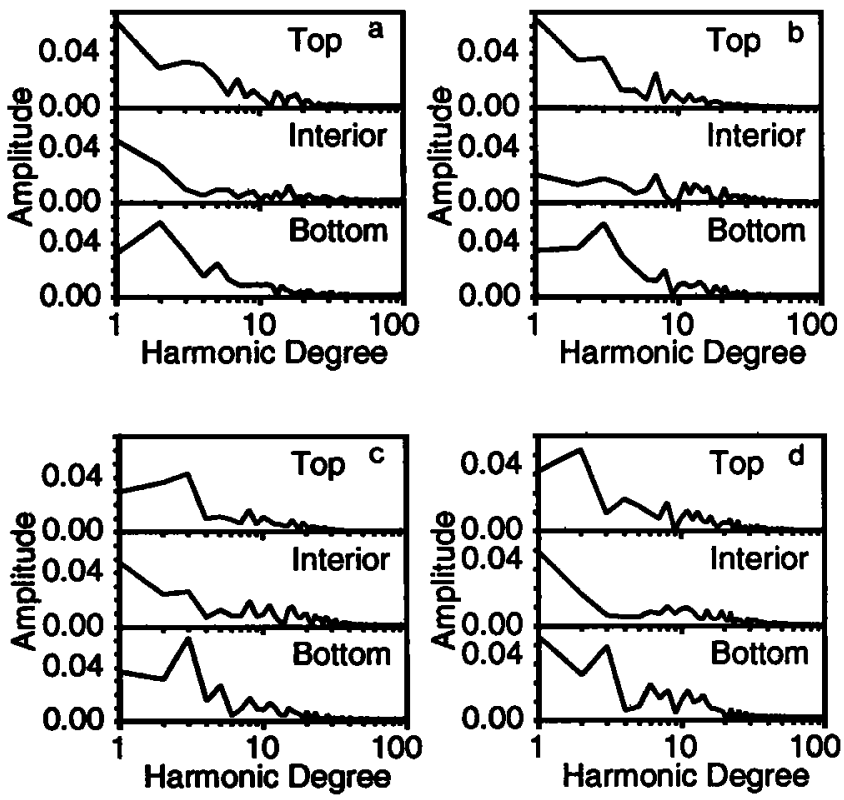

Fig. 6. Corresponding spectra of four frames of temperature in Figure 5 for case 3 . 
in magnitude, but $T_{3}$ and $T_{4}$ increase (Figure 4). The increase in $T_{3}$ reflects the fact that a new plume has been generated beneath the raft (arrow in Figure $5 b$ ). Such variations of $T_{1}, T_{2}$, $T_{3}$, and $T_{4}$, which may also be seen at other times when the raft slows down, lead to an important observation: as the raft velocity decreases, the long-wavelength thermal structure responsible for raft motion tends to break up into relatively shorter-wavelength structure; the resulting thermal structure, due to its shorter wavelength, is unable to greatly effect raft motion. From B to $\mathrm{C}$, the raft is stationary, and due to the less efficient heat transfer through the raft (e.g., Table 1 shows that average heat flux out of the raft is about one quarter of the average heat flux from the surface excluding the raft), heat accumulates beneath the raft, which is evident through the increasing heat flux out of the raft $F$ (Figure 4), and the growing thermal plume under the raft (Figure $5 c$ ). Interestingly, during the heating process while $T_{1}, T_{2}$, and $T_{3}$ increase, $T_{4}$ decreases (Figure 4 ), and similar phenomena occur at other times when long-wavelength structure grows. This suggests that the increasing long-wavelength structure is partially at the expense of relatively shorter wavelength structure (Figure 4). From B to C, $|\vec{M}|$ has no major change, but the direction of $\vec{M}$ rotates $180^{\circ}$, reflecting the fact that the original cold zone beneath the raft has now become hot. As the increasing heat beneath the raft or the increasing large-scale thermal structure $\left(T_{1}\right.$ and $\left.T_{2}\right)$ reaches a critical level, the raft starts to move off the hot zone. The increase in raft velocity after $\mathbf{C}$ enhances the hot zone because of the resulting thermal boundary layer instability, and causes a continuous increase in $T_{1}, T_{2}$, and $|\vec{M}|$ from $\mathrm{C}$ to $\mathrm{D}$ (Figure $5 d$ ) and after. The increasing $T_{1}$ and $T_{2}$ provide additional forces to drive the raft off the hot zone.

The spectra (Figure 6) representing the four time intervals just discussed are dramatically different from the cases with no raft (case 1). Significant long-wavelength anomalies can be observed from the spectra and the shaded temperature (Figure 5). Long-wavelength heterogeneity within thermal boundary layers is also significant (Figure 6) and is comparable in magnitude to that in the interior. However, there is a poor correlation between boundary layer heterogeneity and raft motion. The spectra in the thermal boundary layers reflect the pattem of convection and have significant amplitude at the wavenumber equal to the number of upwelling plumes.

In order to further show the periodicity of raft motion, a Fourier transform is applied to raft velocity for case 3 (i.e., this is a transform in time while previous transforms are in space). The spectra for the whole period of time (dashed line in Figure 7a) shows that predominant periods widely range from about seven to 20 transit times. However, the predominant periods longer than 10 transit times result from the reversal in raft motion which occurs at transit time 50 (Figure 4). Indeed, the spectrum for the period of time excluding the last burst in raft velocity (solid line in Figure 7a) shows that there are two predominant periods at about 10 and seven transit times.

When a significant fraction of internal heating is added to the two cases at a Rayleigh number of $10^{5}$ (cases 5 and 6), we still observed periodic raft motion, a strong correlation between raft motion and large-scale structure $T_{1}$ and $T_{2}$ (Figures 8 and 9), and significant long-wavelength structure within the fluid interior (Figures 8, 9, and 10). We also observed similar physical processes controlling raft motion and the generation of long-wavelength structure, such as consumption of longwavelength structure while raft velocity decreases, as observed
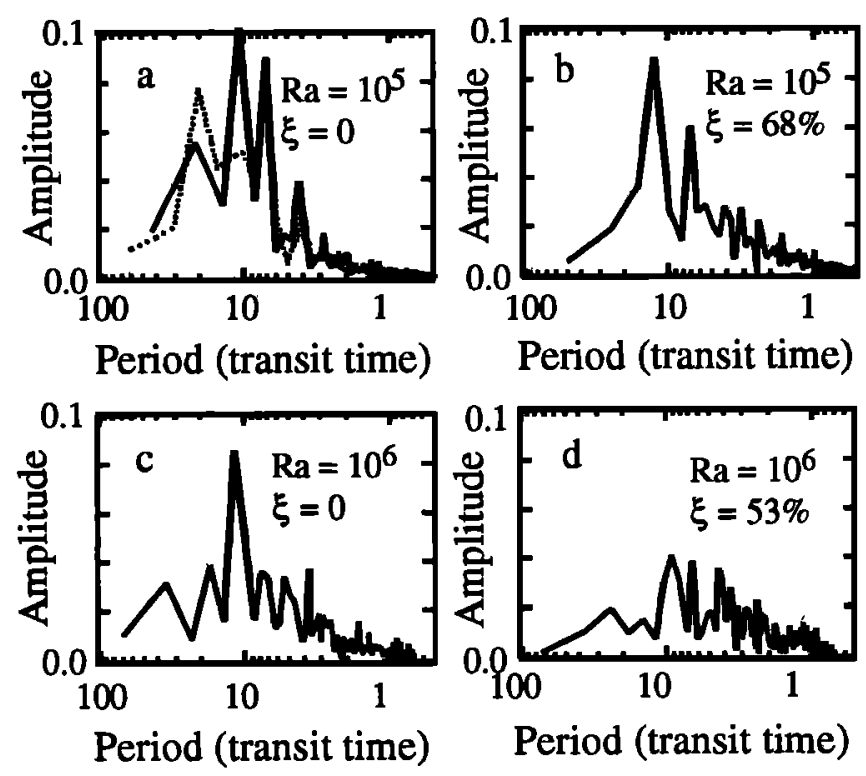

Fig. 7. Spectra of raft velocity for cases 3, 5, 7, and 9 which are shown in Figures $7 a, 7 b, 7 c$, and $7 d$ respectively. (a) solid and dash lines for time from 0.005 to 0.075 (which excludes a strong velocity reversal) and from 0.005 to the end, respectively; $(b)$ for time from 0.01 to the end; $(c)$ for time from 0.003 to the end; (d) for time from 0.003 to the end. The spectra for each case are normalized by their characteristic velocity $\tilde{u}_{\theta}$ (equation (31)). $\xi$ is the percent of internal heating.

in case 3. There are two predominant periods in raft motion for case 5 (Figure $7 b$ ) at about 12 transit times and at seven transit times, and for case 6 the predominant period is about 9 transit times. However, several important differences from the bottom heated cases can be observed. First, the duration of negligible raft motion decreases and secondary raft motion occurs frequently. Second, the correlation between raft motion and long-wavelength thermal structure becomes complicated. While for the case with a large raft (case 5), $T_{3}$ and $T_{4}$ are not correlated with raft motion (Figure 8 ), for the small raft case (case 6), some peaks in $T_{3}$ seem to coincide with bursts in raft motion (Figure 9).

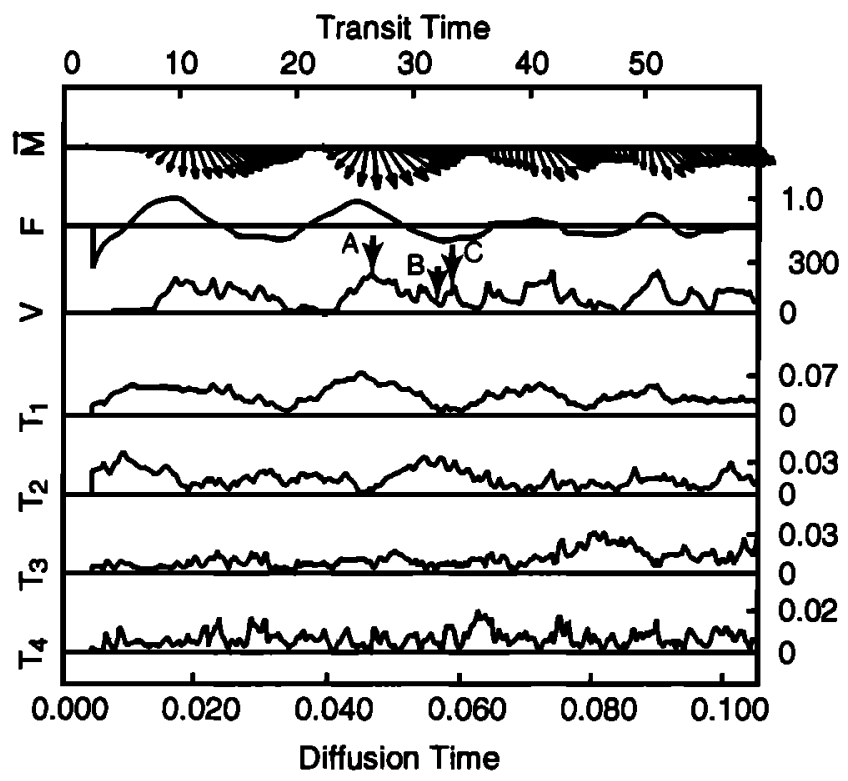

Fig. 8. Time history for case 5. (See Figure 4 for detail.) 


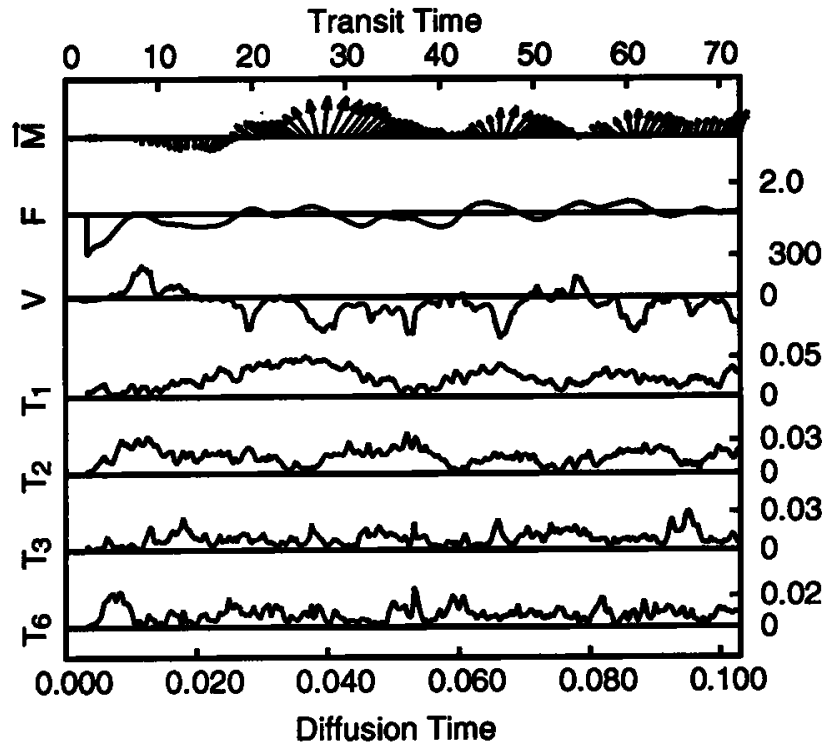

Fig. 9. Time history for case 6. (See Figure 4 for detail.)

Significant amount of internal heating in these two cases (the averaged internal heating rates are $68 \%$ and $63 \%$ for cases 5 and 6, respectively) may account for these differences. Internal heating results in frequent occurrences of instabilities from the lower thermal boundary [Weinstein et al., 1989]. These instabilities from the bottom boundary substantially enhance the accumulation of heat beneath the raft while the raft is stationary and cause the duration of periods of negligible raft motion to decrease. These instabilities, when forming thermal structure of a wavelength larger than raft size, may also result in secondary peaks in raft motion. This is well illustrated in Figure 10 (the position of these frames in the overall time history are labeled in Figure 8), and the animation
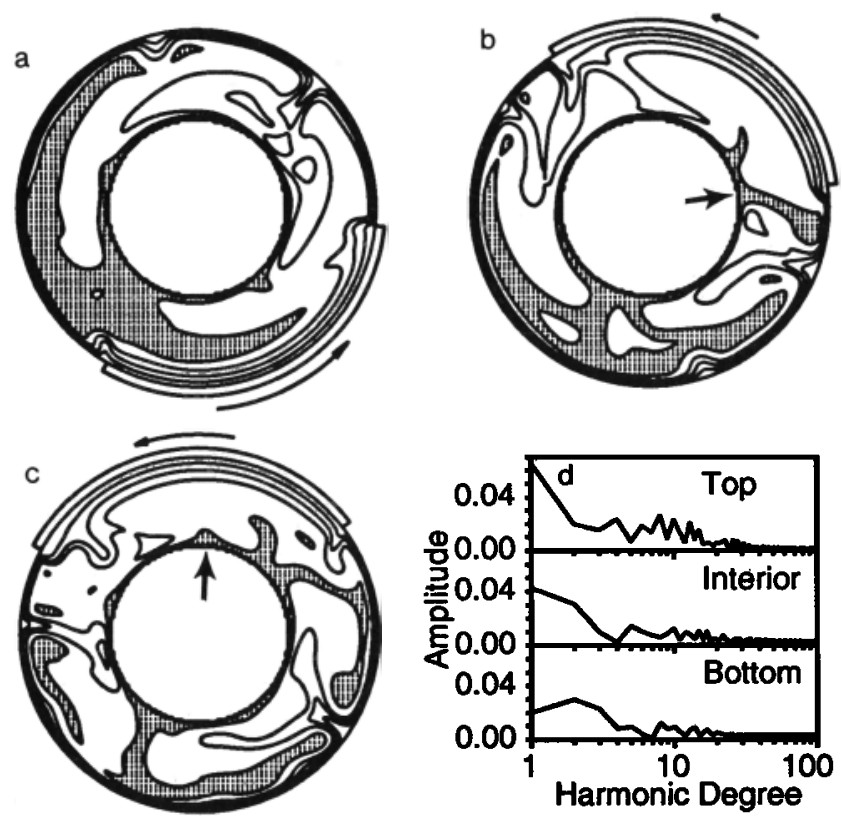

Fig. 10. Temperature field (Figures 10a-10c) for three instants labeled in Figure 8 for case 5; Figure $10 d$ is the spectra for the temperature field in Figure 10b. In Figures 10a-10c, the shaded regions have a temperature greater than 0.8 . of the model is on the video tape. At $A, T_{1}$ is dominant over all other scales, and the raft moves off a hot plume (Figure 10a). From $A$ to $B$, as the raft moves away from the hot plume and slows down, the hot plume decays and a new one forms behind the raft (Figure 10b), which is also evident through the decreasing $T_{1}$ and growing $T_{2}$ (Figure 8). The newly generated plume succeeds the original main plume to drive the moving raft and causes a secondary peak in raft velocity. After the secondary peak decays (Figure 8), another new plume forms beneath the raft (Figure 10c) which eventually causes another secondary peak in the raft velocity. For case 6 with a small raft, thermal structure $T_{3}$ seems to be able to produce significant raft motion, but $T_{6}$ is unable to do so (Figure 9). This again suggests that raft motion is mainly controlled by thermal structure with a wavelength larger than raft sizes. However, because variations of thermal structure of a shorter wavelength usually have a shorter period than raft velocity and a smaller magnitude than $T_{1}$ and $T_{2}$ (e.8., $T_{3}$ in Figure 9), it is often difficult to analyze the relationship between short wavelength structure and raft motion.

The long-wavelength structure is evident from both the shaded temperature field (Figure 10b) and the corresponding spectra (Figure 10d). The two hot zones (Figure 10b) are responsible for the significant amplitude at degree 2 in both the bottom boundary layer and the fluid interior. The imbalance of the two hot zones in both strength and location causes significant amplitude at degree 1 which, in the top boundary layer, is dominant over degree 2.

The internal heating rate is higher for the case with the larger raft because the larger raft results in a higher mean intemal temperature (Table 1 ) which stifles the flow of heat into the lower boundary. Neither the kinetic energy nor the raft velocity increase after a significant amount of internal heating is introduced.

\subsection{Cases With Rayleigh Number $10^{6}$}

Periodic raft motion, significant long-wavelength thermal structure, and a correlation between the long-wavelength structure and raft motion are still observed when the Rayleigh number is increased from $10^{5}$ to $10^{6}$ (Figures 11 and 12 for

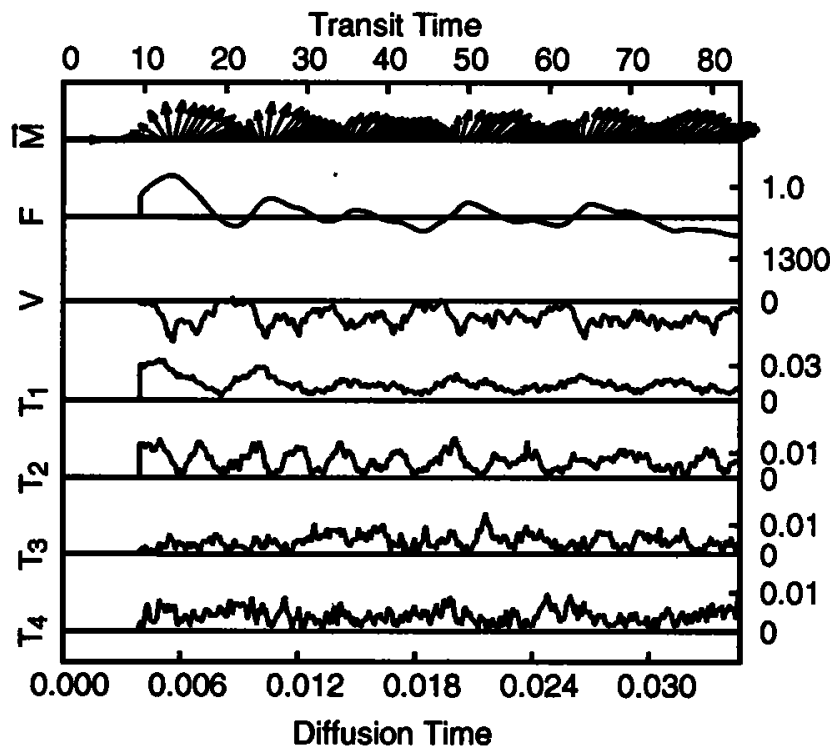

Fig. 11. Time history for case 7. (See Figure 4 for detail.) 


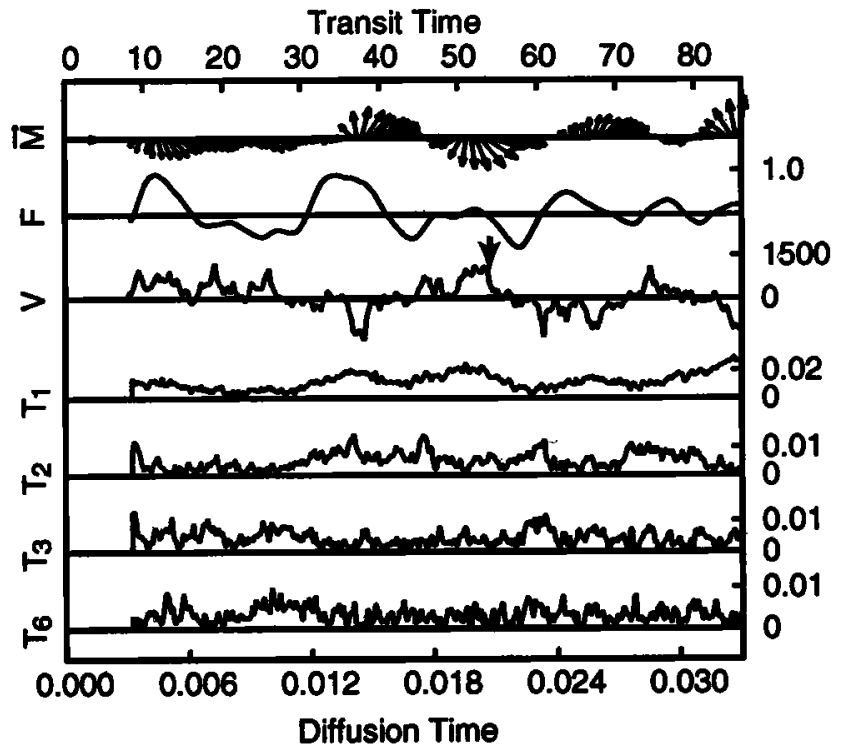

Fig. 12. Time history for case 8. (See Figure 4 for detail.)

cases 7 and 8 and Figure 13b). Compared to the bottom heated cases at $R a=10^{5}$ (cases 3 and 4), periods between each burst of raft motion decrease substantially in terms of the diffusion time, but the predominant periods in terms of the transit time only change slightly. There is only one predominant period at about 11 transit times for case 7 (Figure 7c), compared to 10 and seven transit times for case 3 at $R a=10^{5}$ (solid line in Figure 7a). For the case with a small raft (case 8), the raft becomes stationary more frequently (Figure 12) compared to the large raft case (Figure 11); the predominant period at about 12 transit times is close to the dominant period for case 7. For both cases 7 and 8 , raft motion is mainly controlled by $T_{1}$ and
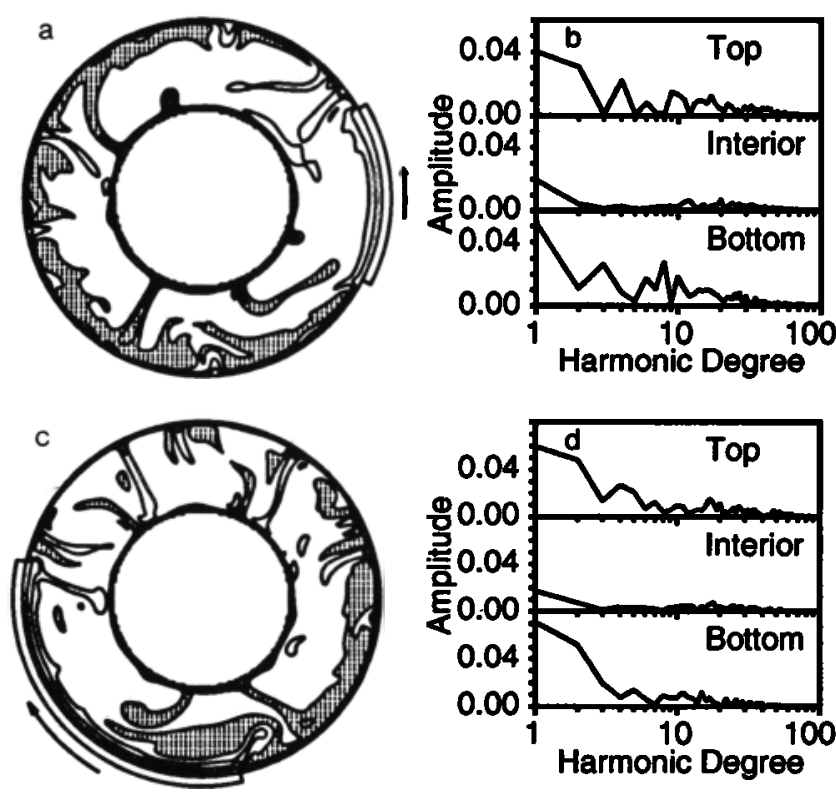

Fig. 13. Representative temperature fields and their corresponding spectra. (a) temperature field for case 8 at the time labeled in Figure 12; (b) spectra of temperature field in Figure 13a; (c) temperature field for case 9 at the time labeled in Figure 14; (d) spectra of temperature field in Figure 13c. In Figures 13a and 13c, the shaded regions have a temperature greater than 0.428 and 0.667 , respectively.
$T_{2}$, but for the small raft case, some bursts in raft motion are clearly caused by $T_{3}$ (e.g., the peaks of $V$ at transit time 20 in Figure 12), which confirms that raft motion is controlled by thermal structure with a wavelength larger than the raft size.

When significant internal heating is introduced into two cuses at $R a=10^{6}$ (cases 9 and 10 have 53\% and 50\% internal heating, respectively), raft motion becomes complicated, and we observe no periods of raft stationarity for the larger raft (Figure 14 for case 9). However, two predominant periods at about 10 and 5 transit times (Figure $7 d$ ) are still evident for case 9, although now the spectrum of raft velocity is much more broadly distributed compared to both lower Rayleigh number cases and cases with no internal heating. The irregular raft motion is also due to the thermal boundary layer instability: both internal heating and high Rayleigh number enhance these instabilities. For case 9, raft motion is mainly controlled by $T_{1}$, but $T_{2}$ is also responsible for some bursts in raft motion (e.g., the burst at transit time 63 in Figure 14). Interestingly, the relative position between the raft and the large-scale cold zones has negligible variation as the raft moves ( $\bar{M}$ in Figure 14); this means that a hot zone constantly lags directly behind the raft and drives its motion. The slight changes in the direction of $\bar{M}$ are often coincident with the largest fluctuations in raft velocity and $T_{1}$.

The temperature field and its spectra for cases at $R a=10^{6}$ (two representative frames for cases 8 and 9 are presented in Figure 13) show that significant long-wavelength thermal structure exists within both the thermal boundary layers and the fluid interior. Within the fluid interior, the longest wavelength structure, $T_{1}$, is predominant over other wavelength structure (Figures $13 b$ and 13d). There are a significant number of small-scale thermal anomalies. But the small-scale thermal anomalies are not evenly distributed in the fluid layer, thus causing significant long-wavelength structure.

Depth dependence of RMS of the representative temperature field for the case with significant amount of internal heating (case 9) differs from that for the case with no internal heating (case 8) (Figure 3); in fact, the latter has less heterogeneity in the top boundary layer.

\section{Discussion}

With no raft on the surface, convection either reaches a steady-state with regular convection cells at $R a=10^{5}$ (case 1) or becomes intermittent at the higher $R a$ (case 2). The spectra (Figures $2 b$ and $2 d$ ) show that the thermal structure is characterized by short-wavelength anomalies. Interestingly, for case 2, significant long-wavelength structure only occurs within the bottom thermal boundary layer (Figure $2 d$ ). Considering that models in a Cartesian geometry [Jarvis and Peltier, 1986] usually yield identically significant longwavelength structure within both the top and bottom boundary layers, this shows that a difference in geometry may result in a significant difference in thermal structure. After introducing a continent like raft, significant long-wavelength thermal anomalies are generated through the whole fluid layer. In addition, raft motion appears periodic.

The long-wavelength thermal anomalies may be related to the observed large-scale seismic heterogeneity. Considering that convection models without rafts yield less internal longwavelength structure [Machetel, 1990], e.g., cases 1 and 2, we suggest that the continent like rafts, which are an imposed large-scale heterogeneity on the surface, may play an 


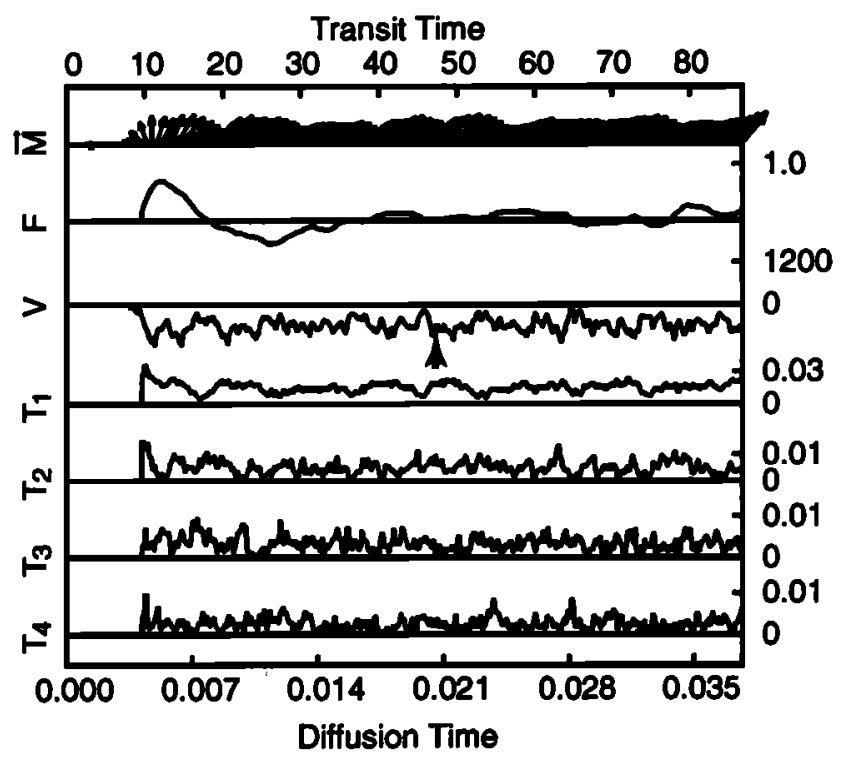

Fig. 14. Time history for case 9. (See Figure 4 for detail.)

important role in mantle convection. Comparison of the depth dependence of RMS of temperature between case 9 and cases 2 and 8 (Figure 3) suggests that with the parameters studied here, a model with internal heating and a nonsubducting raft is capable of producing more heterogeneity in the top thermal boundary layer. While the spectra in the top boundary layer for case $9(W / D=4)$ decrease with wavenumbers $k$, with an approximated function of $\boldsymbol{k}^{-1}$, such a relationship between the spectra and wavenumbers is not observed for case 2 without a raft (Figure 15). Both the more heterogeneity within the top boundary layer and the $k^{-1}$ relationship between the spectra and wavenumber $k$ from case 9 seem to be compatible with the seismic observations as reviewed before [Gudmundsson, 1989; Tanimoto, 1990b; Zhang and Tanimoto, 1991]. Since the $k^{-1}$ relationship is observed for the case with a raft (case 9), this also supports the explanation made by Tanimoto [1990b] that the seismically observed $k^{-1}$ may result from the presence of continents. However, it should be pointed out that the decrease of spectra with wavenumbers becomes gentle within the fluid interior in our models.

The spectra within thermal boundary layers and the interior may substantially differ in both amplitude and dependence on wavenumber. While the amplitude of spectra in thermal boundary layers appears to be insensitive to Rayleigh number.
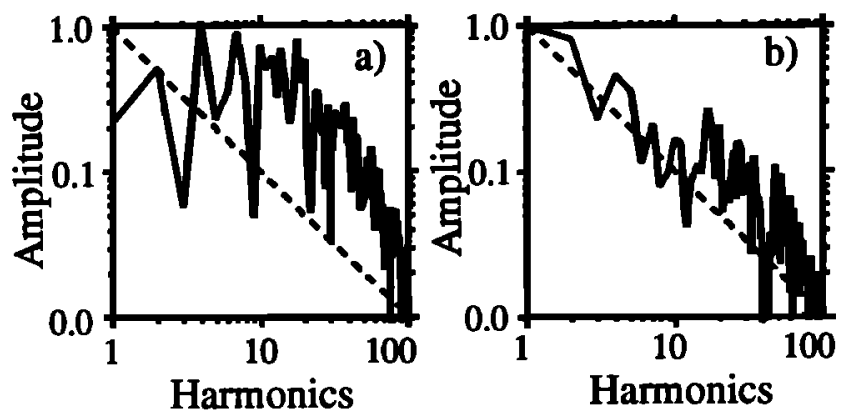

Fig. 15. Dependence of spectre of the top thermal boundary layen on wavenumbers. (a) for temperature field in Figure $2 c$ (case 2); (b) for temperature field in Figure $13 c$ (case 9). the amplitude in the fluid interior decreases as Rayleigh number is increased (Figures $13 b$ and 13d). However, the longwavelength structure including the degree one remains significant compared to short-wavelength structure, even as the Rayleigh number is increased to $10^{6}$ (Figures $13 b$ and $13 d$ ). Indeed, the significant $T_{1}$ and $T_{2}$ control raft motion.

The continent like raft is responsible for the generation of long-wavelength structure and the generated thermal structure at a wavelength larger than raft size in turn controls raft motion. With a high viscosity raft, sluggish conduction is the only form of heat transfer possible through the raft, which is evident through the fact that heat flux out of the raft is much smaller than global heat flux (Table 1). As a result, heat accumulates beneath the raft. The accumulated heat causes long-wavelength thermal heterogeneity and drives raft motion. The moving raft releases the accumulated heat and this in turm leads to a decrease in raft velocity. The end results are a periodic raft velocity and a strong correlation between raft motion and long-wavelength thermal structure.

The mechanism of raft motion is better understood by looking in detail at the termination and initiation of a burst in raft motion. An upwelling plume is usually behind a moving raft. As the moving raft approaches another upwelling plume, the raft velocity will decrease and even stop if the approached upwelling plume is comparable in magnitude to the upwelling plume behind the raft. The raft may stop over a downwelling or an upwelling plume, when the raft becomes surrounded by either two upwelling or two downwelling plumes. During the period of negligible raft velocity, heat accumulates beneath the raft. The accumulated heat causes the overridden upwelling plume (or downwelling plume) to enhance (or diminish) and eventually causes the raft to move. Usually, while the raft is moving, bottom boundary layer instabilities pump additional heat into the high temperature area beneath the raft by the collision of smaller plumes into larger ones; this causes a significant change in global thermal structure and the raft velocity (see animation for case 3 in the video tape). This feeding of small thermal anomalies into preexisting largescale structure was previously shown in isoviscous, high Rayleigh number convection [Christensen, 1987; Hansen and Ebel, 1989]. Indeed, in the terminology of Vincent and Yuen [1988], the preexisting large-scale thermal structure could be called a thermal attractor.

Although predominant periods of the periodic raft motion vary greatly with the Rayleigh number and internal heating in terms of diffusion time, they change only slightly in terms of transit time (Figure 7). Considering that raft motion is controlled by long-wavelength thermal structure within the fluid, the predominant periods have clear physical meaning, the period during which the fluid system forms thermal anomalies capable of causing raft motion and releases the thermal anomalies. Perhaps of more importance is that for all the cases shown in Figure 7 there is a significant (often dominant) period at about 10 transit times. For a small raft, the motion has a similar primary period: 9 and 12 transit times for cases 6 and 8, respectively. Since this primary period appears to be relatively insensitive to Rayleigh number, the amount of internal heating, and raft sizes, we may scale it to Earth. With a plate velocity $5 \mathrm{~cm} / \mathrm{yr}$, the transit time for the Earth is about $60 \mathrm{~m} . y$. for whole mantle convection, suggesting a scaled period of about $600 \mathrm{~m} . y$. Although caution must be exercised, the predominant period of supercontinent aggregation may be close to 500 m.y. [Hoffman, 1992]. We do not investigate 
aggregation and breakup of the nonsubducting raft in this paper, which would involve complicated multiplate's effects [Gurnis, 1988]; however, the fundamental mechanism causing periodic raft motion from our models would remain the same.

\section{Conclusion}

In a model with a cylindrical annular geometry we have introduced a continent like raft into a system of thermal convection. Significant long-wavelength thermal structure is generated not only within the bottom and top boundary layers but also within the fluid interior. The existence of significant long-wavelength thermal structure over a wide range of parameters suggests that such phenomena may be fundamental for Earth. While the magnitude of the spectra of longwavelength thermal structure within the fluid interior decreases as Rayleigh number is increased, the relative importance of this structure compared to higher harmonics is still significant. The magnitude of spectra within the boundary layers is insensitive to the Rayleigh number and is always significant. The magnitude of spectra within the top thermal boundary layers decreases with wavenumbers $k$, with an approximated function of $k^{-1}$, and with a certain amount of internal heating, the RMS within top thermal boundary layers is much larger than that within the bottom thermal boundary layers, both of which are compatible with seismic observations.

Raft motion appears periodic, although the details of raft motion depend on Rayleigh number, internal heating, and raft size. Raft motion with a period about ten transit times is usually significant for most cases (Figure 7). This period scales to about $600 \mathrm{~m} . y$., close to the $300-500$ m.y. period suggested for supercontinent aggregation and dispersal. Raft motion is controlled by thermal structure of a wavelength larger than raft size, but the structure with the longest wavelength, such as $T_{1}$ and $T_{2}$, usually has a much greater effect on raft motion than smaller-scale structure.

Both significant long-wavelength structure and periodic raft motion are consequences of the dynamic interaction between the raft and convection. When the raft is relatively stationary, heat accumulates beneath due to the less efficient heat transfer through the raft and instabilities from the bottom boundary layer, thus causing long-wavelength thermal structure and enhancing raft motion. Accompanying the enhanced raft movement, the long-wavelength thermal anomalies diminish, and the raft velocity is decreased.

Although these dynamic models are still ideal, they may explain observables such as long-wavelength seismic heterogeneity in the mantle and possibly the periodicity of supercontinent aggregation and dispersal. Considering that models without rafts generate less long-wavelength structure, it is suggested that continent like rafts play an important role in mantle convection and should be properly taken into account.

\section{Appandix A: Comparison Writ A LinEar Stabiuty ANalysis}

A proper approach to verify a computer code is to compare a solution from the code with that from an analytical method for a problem which is fully described by the governing equations. For our present problem, we choose the critical Rayleigh number, which is a measure of stability of a fluid system and can be obtained from a linear stability analysis (LSA). The linear stability analyses in spherical shell geometry and
Cartesian geometry have been well documented [Jeffreys and Bland, 1951; Chandrasekhar, 1961]. A similar technique is adopted here for an annulus in a cylindrical coordinate system.

Consider a annulus of incompressible fluid subject to a constant radial gravitational field 8 . Because the present purpose is to verify our code, only bottom heating, isothermal and free-slip boundary conditions are considered. The perturbation equations may be written in the forms [Chandrasekhar, 1961]

$$
\begin{gathered}
v \frac{\partial^{2} u_{i}}{\partial x_{j} \partial x_{j}}-\frac{\partial}{\partial x_{i}}\left(\frac{\delta p}{p}\right)+\alpha \theta_{g} \frac{x_{i}}{r}=0, \\
\frac{\partial u_{i}}{\partial x_{i}}=0 \\
\frac{\partial \theta}{\partial t}+\beta(r) u_{i} x_{i}=\kappa \frac{\partial^{2} \theta}{\partial x_{j} \partial x_{j}}
\end{gathered}
$$

where $\theta$ and $\delta p$ are the perturbations in the temperature and the pressure, respectively; $v$ is the kinematic viscosity; for the geometry and boundary conditions in our model,

$$
\beta(r)=\frac{T_{o}-T_{i}}{r^{2} \ln \left(R_{o} / R_{i n}\right)},
$$

where $R_{i n}$ is the inner radius, and all other symbols have the same definitions as previous ones in the text.

After applying boundary condition to above equations and long mathematical derivation, we obtain the secular equation:

$$
\left\|P_{i j}+m^{2} \frac{R_{o}^{3}}{\left(R_{0}-R_{i n}\right)^{3}} \cdot \ln \left(\frac{R_{o}}{R_{i n}}\right) R a \cdot\left(\frac{N_{m_{0} j}}{k_{j}^{4}} \delta_{i j}+Q_{i j}\right)\right\|=0 \text {. }
$$

where

$$
\begin{aligned}
P_{i j} & =\frac{1}{k_{j}^{3}} \int_{k_{j} j}^{k_{j}} S_{m}\left(\frac{k_{i} x}{k_{j}}\right)\left[2 x^{3} S_{m-1}(x)\right. \\
+ & \left.\left(x^{2}-2 m x^{2}-x^{4}\right) S_{m}(x)\right] d x, \\
N_{m, j} & =\frac{1}{2}\left\{\left[S_{m}^{\prime}\left(k_{j}\right)\right]^{2}-\left[\zeta S_{m}^{\prime}\left(k_{j} \zeta\right)\right]^{2}\right\}, \\
Q_{i j} & =\frac{2}{k_{i}^{3} k_{j}^{3} m}\left[\frac{m+1}{1-\zeta^{2 m+2}} T_{m, i} T_{m, j}\right. \\
& \left.+\frac{m-1}{1-\zeta^{-2 m+2}} Z_{m, i} Z_{m, j}\right],
\end{aligned}
$$

$\delta_{i j}$ is kronecker delta; $m$ is the wavenumber; $R a$ is the Rayleigh number defined before; $\left\|A_{i j}\right\|$ represents the determinant of any matrix $A_{i j} ; \zeta$ is the ratio of the inner radius to the outer radius; $k_{j}$ is eigenvalue, i.e., the solution of eigenequation $S_{m}\left(k_{j} \zeta\right)=0$, and $S_{m}\left(k_{j} r\right)$ is defined as

$$
S_{m}\left(k_{j} r\right)=Y_{m}\left(k_{j} \zeta\right) J_{m}\left(k_{j} r\right)-J_{m}\left(k_{j} \zeta\right) Y_{m}\left(k_{j} r\right) \text {. }
$$

where $J_{m}(x)$ and $Y_{m}(x)$ are the Bessel functions of the first and second kind, respectively, both of order $m$. In (A8), $T_{m, i}$ and $Z_{m, i}$ are defined as

$$
T_{m, i}=S_{m}^{\prime}\left(k_{i}\right)-\zeta^{m+1} S_{m}^{\prime}\left(k_{i} \zeta\right) .
$$


TABLE A1. Critical Rayleigh Numbers from Linear Stability Analysis and the Finite Element Code

\begin{tabular}{lcccccccc}
\hline & \multicolumn{8}{c}{ Wavenumber $m$} \\
\cline { 2 - 9 } & 1 & 2 & 3 & 4 & 5 & 6 & 7 & \multicolumn{1}{c}{8} \\
\hline $\operatorname{Re}$ (LSA) & 1380 & 921 & 691 & 718 & 874 & 1146 & 1549 & 2109 \\
$\operatorname{Ra}$ (the code) & 2541 & 908 & 683 & 711 & 866 & 1136 & 1539 & 2100 \\
Error, \% & - & 1.4 & 1.2 & 1.0 & 0.9 & 0.9 & 0.7 & 0.4 \\
\hline
\end{tabular}

$$
Z_{m, i}=S_{m}^{\prime}\left(k_{i}\right)-\zeta^{-m+1} S_{m}^{\prime}\left(k_{i} \zeta\right)
$$

where

$$
S_{m}^{\prime}(a)=\left.\frac{d S_{m}(x)}{d x}\right|_{x=a}
$$

The secular equation is solved in the second-order approximation; that is, only first two eigenvalues $k_{1}$ and $k_{2}$ are taken into account, which is sufficiently accurate. For different wavenumber $m$, the critical Rayleigh numbers are computed and shown in Table A1.

Critical Rayleigh number can also be numerically determined by following approach. A kinetic energy of flow is defined as

$$
E=\int_{S}\left(u_{r}^{2}+u_{\theta}^{2}\right) d S^{\prime},
$$

where the integrated domain $S$ is the whole fluid layer. Given a Rayleigh number and a wavenumber of initial perturbation temperature, we calculate $E$ at every time step. By monitoring the kinetic energy $E$ variation with time and changing the Rayleigh number, we can find a Rayleigh number at which $E$ remains constant with time. Obviously, this Rayleigh number is just the critical Rayleigh number for the given perturbation wavenumber.

Table A1 presents the comparison of critical Rayleigh numbers from the two different approaches for eight different initial wavenumbers. The critical Rayleigh numbers from the code are consistent with those from the linear stability analysis except when the initial wavenumber is equal to 1 . The remarkable discrepancy for $m=1$ may be due to numerical error. It has been found that a stable two cell convection mode which corresponds to $m=1$ can not be numerically generated in the annulus and that there exist 6 cells when $R a=2541$ for $m=1$, which means that mode $m=1$ switches to mode $m=3$.

\section{APPENDIX B: VIDEO}

Animations ${ }^{1}$ of the temperature fields, spectra, and raft velocity for cases 3-10 (Table B1) have been recorded on video. The frames which make up the video are each separated by 20 computational time steps. For each case, an animation has included sufficient frames such that at least a complete period, during which significant long-wavelength thermal

\footnotetext{
${ }^{1}$ Animations are available on videotape (VHS), product code 3JB0193V: \$25.00. Orders must be prepaid by check, money order, or credit card. AGU accepts American Express, VISA, and MasterCard. Credit card orders may be placed through Kosmos or by calling 800 . 966-2481 toll free or 202-462-6900 in Washington, D.C. or outside the United States. Orders placed by mail should be addressed to AGU Caging, 2000 Florida Avenue, N.W., Washington, DC 20009; Fax: 202. 328-0566; Electronic mail: Kosmos: CUST_SER@PINET.AIP.ORG
}

TABLE B1. Time Period of the Animation for Each Case and Sequence of Animation on the Accompanying Video

\begin{tabular}{cccc}
\hline Case & Start Tme & End Time & Sequence on Video \\
\hline 3 & 0.0385 & 0.0602 & 1 \\
4 & 0.0271 & 0.0479 & 2 \\
5 & 0.0376 & 0.0602 & 3 \\
6 & 0.0620 & 0.0770 & 4 \\
7 & 0.0175 & 0.0258 & 5 \\
8 & 0.0189 & 0.0284 & 6 \\
9 & 0.0193 & 0.0304 & 7 \\
10 & 0.0234 & 0.0331 & 8 \\
\hline
\end{tabular}

anomalies are formed and released accompanying a increase in raft motion, is shown.

Acknowledgments. We would like to thank B. Hager, C. Jaupart, and an anonymous reviewer for their detail reviews. Funded by NSF grants EAR-8957164 and EAR-8904660 and The David and Lucile Packard Foundation.

\section{REFERENCES}

Anderson D. L., Hotspots, polar wander, Mesozoic convection and the geoid, Nature. 297, 391-393, 1982.

Bond, G. C., and M. A. Kominz, Construction of tectonics subsidence curves for the early Paleozoic miogeocline, southem Canadian Rocky Mountains: Implications for subsidence mechanisms, age of breakup, and crustal thinning, Geol_Soc. Am. Bull, 25, 155-173, 1984.

Brooks, A. N., A Petrov-Galerkin finite element formulation for convection dominated flows, Ph.D. thesis, Calif. Inst. of Technol., Pasadena, 1981.

Chandrasekhar, S., Hydrodynamic and Hydromaeneric Stabjlity, 220 pp., Dover, New York, 1961.

Christensen, U. R., Time-dependent convection in elongated RayleighBenard cells, Geophys. Res.Leth, 14, 220-223, 1987.

Dalziel, I. W. D., Pacific margins of Laurentia and East AntarcticaAustralia as a conjugate rift pair: Evidence and implications for an Eocambrian supercontinent, Geology 19, 598-601, 1991.

Davies, G. F. Mantle convection under simulated plates: Effects of heating modes and ridge and trench migration, and implications for the core-mantle boundary, bathymetry, the geoid and Benioff zones, Geophys.J.R. Astron. Soc. 84, 153-183, 1986.

Davies, G. F., Role of the lithosphere in mantle convection, J. Geophys. Bes. $23,10,451-10,466,1988 a$.

Davies, G. F., Oceanic bathymetry and mantle convection, 1, Largescale flow and hotspots, J. Geophys. Res., 93 10,467-10,480, 1988b.

Davies, G. F., Mantle convection model with a dynamic plate: Topography, heat flow and gravity anomalies, Geophys. J. 98, 461464, 1989.

Davies, J. H., Some problems in mantle structure and dynamics, Ph.D. thesis, Calif. Inst. of Technol., Pasadena, 1990.

Dziewonski, A. M., Mapping the lower mantle: Determination of lateral heterogeneity in P velocity up to degree and order 6. L. Geophys. Res., 89, 5929-5952, 1984.

Driewonski, A. M., B. H. Hager, and R. J. O'Connell, Large-scale heterogeneities in the lower mantle. I. Geophys. Res., 82, 239-255, 1977.

Elder, J., The Bowels of the Earth, 222 pp., Oxford University Press, New York, 1976

Gudmundsson, O., Some problems in global tomography, Ph.D. thesis, Calif. Inst. of Technol., Pasadena, 1989.

Gumis, M., Large-scale mantle convection and the aggregation and dispersal of supercontinents, Nature, 332, 695-699, 1988.

Gumis, M., and B. H. Hager, Controls on the structure of subducted slabs, Nature, 335, 317-322, 1988.

Gumis, M., and S. Zhong, Generation of long-wavelength wavelength heterogeneity in the mantle by the dynamic interaction between plates and convection, Geophys. Res. Lett. 18. 581-584, 1991.

Hager, B. H., and R. W. Clayton, Constraints on the structure of mantle convection using seismic observations, flow models, and the geoid, in Mantle Convection edited by R. W. Peltier, pp. 657-763, Gordon and Breach, New York, 1989. 
Hansen, U., and A. Ebel, Time-dependent thermal convection--A possible explanation for a multiscale flow in the Earth's mantle, Geophys. J. 94, 181-191, 1989.

Hoffman, P. F., Did the breakout of Lauentia tum Gondwanaland insideout?, Science, 252, 1409-1412, 1991.

Hoffman, P. F., "Supercontinents", in Encyclopedia of Earth System Science, Vol. 4, edited by W. A. Nierenberg, pp. 323-328, Academic, San Diego, Calif., 1992.

Ho-Liu, P., B. H. Hager, and A. Raefsky, An improved method of Nusselt number calculation, Geophys. J. R. Astron. Soc. 88, 205-215, 1987.

Honda, S., The RMS residual temperature in the convecting mantle and seismic heterogeneiries, J. Phys. Farh. 35, 195-207, 1987.

Hughes, T. J. R., The Finite Element_Method.631 Pp., Prentice-Hall, Englewood Cliffs, N. J., 1987.

Inoue, H., Y. Fukao, K. Tanabe, and Y. Ogata, Whole mantle P-wave travel time tomography, Phys. Earth Planet. Inter. 59, 294-328, 1990.

Jarvis, G. T., and W. R. Peltier, Mantle convection as a boundary layer phencmenon, Gecohys. J.R. Astron. Soc., 68, 389-427, 1982.

Jarvis, G. T., and W. R. Peltier, Lateral heterogeneity in the convecting mantle J.Geophys. Res. 21, 435-451, 1986.

Jarvis, G. T., and W. R. Peltier, Low-wavenumber signatures of timedependent mentle convection, Phys. Farth Planet. Inter. 59, 182-194, 1990.

Jeffreys, H., and M. E. M. Bland, The instability of a fluid sphere heated within, Mon. Not. R.Astron. Soc. London Geophys. Supol. 6. 148$158,1951$.

King, S. D., and B. H. Hager, The relationship between plate velocity and trench viscosity in Newtonian and power-law subduction calculations, Geophys. Res. Lett., 17 2409-2412, 1990.

King, S. D., A. Raefsky, and B. H. Hager, ConMan: Vectorizing a finite element code for incompressible two-dimensional convection in the Earth's mantle, Phys. Earth Panet. Inter. 59, 195-207, 1990.

Machetel, P., Short-wavelength lower mantle seismic velocity anomalies, Gecohys. Res. Leth, 17 1145-1148, 1990.

MeKenzie, D. P., J. M. Roberts, and N. O. Weiss, Convection in the Earth's mantle: Towards a numerical simulation, J. Elvid Mech. 62. 4655-538, 1974.

Miller, J. D., and D. V. Kent, Paleomegnetism of the Silurian-Devonian Andreas redbeds: Evidence for an Early Devonian supercontinent? Goolory, 16, 195-198, 1988.

Ricand, $\mathbf{Y}$., and C. Vigny, Mantle dynamics with induced plate tectonics,
I. Geophy. Rex. 94, 17,543-17,559, 1989.

Smith, A. G., A. M. Hurley, and J. C. Briden, Phanerozoic Palaeocontinental Wordd Maps, Combridge University Press, New York, 1981.

Su, W.-J., and A. M., Driewongki, Predominance of long-wavelength heterogeneity in the mantle, Nature, 352, 121-126, 1991.

Tanimoto, T., Long-wavelength S-wave velocity structure throughout the mantle, Gecohys.J. Int 100, 327-336, 1990a.

Tanimoto, T., Predominance of large-scale heterogeneity and the shift of velocity ancmalies between the upper and lower mantle, $\underline{\mathrm{J}}$. Phys. Enth, 38, 493-509, 19906.

Van der Voo, R., Paleozoic paleogeography of North America, Gondwana, and the intervening displaced terranes: Comparisons of paleomagnetism with paleoclimatology and biogeographical pattems, Geol. Soc. Am. Bull. 100, 311-314, 1988.

Van der Voo, R., C. McCabe, and C. R. Scotese, Was Laurentia part of an Eocambrian supercontinent?, in Plate Reconstruction From Paleozoic Paleomagnetism, Geodyn. Ser., Vol. 12, edited by R. Van Der Voo, C. R. Scotese, and N. Bonhommet, Pp. 131-136, AGU, Washington, D.C., 1984.

Vincent, A. P., and D. A. Yuen, Thermal attrator in chaotic convection with high Prandul number fluids, Phys. Rev. A. 38, 328-334, 1988.

Weinstein, S. A., P. L. Olson, and D. A. Yuen, Time-dependent large aspect-ratio themal convection in the Earth's mantle, Geophys. Antrohys. Finid Dyn. 47 157-197, 1989.

Woodward R. L. and G. Masters, Lower-mantle structure from ScS-S differential travel times, Nature, 352, 231-233, 1991.

Zhang, Y.S., and T. Tanimoto, Global Love wave phase velovity variation and its significance to place tectonics, Bhys. Farth Planet. Inter., 66, 160-202, 1991.

M. Gumis and S. Zhong, Department of Geological Sciences, The University of Michigan, 1006 C. C. Little Bldg., Ann Atbor, MI 481091063.
Received September 9, 1991; revised December 10, 1992; accepted December 16, 1992.) 